\title{
A NEW METHOD FOR RECOGNITION AND OBSTACLE DETECTION FOR VISUALLY CHALLENGED USING SMART GLASSES POWERED WITH RASPBERRY PI
}

\author{
K. Sundar Srinivas \\ Asst. Professor \\ Department Of Electronics And Communication Engineering \\ Potti Sriramulu Chalavadi Mallikarjuna Rao College Of Engineering \& Technology, Vijayawada \\ K. Sahithya, G. Lakshmi Tejaswi, K. Hari Gopal, B. Pavan Karthik \\ Student \\ Department of Electronics and Communication Engineering \\ Potti Sriramulu Chalavadi Mallikarjuna Rao College Of Engineering \& Technology, Vijayawada
}

\begin{abstract}
In this paper, we are presenting an electronic device for obstacle detection and face recognition to assist visually challenged users in social interactions. Blind people have low vision to recognize people in the society and this device helps the user to walk without colliding with any obstacles in the path. The device is in the form of smart glasses that has ultrasonic sensor, pi camera and raspberry pi installed on it. The ultrasonic sensors are connected to the raspberry pi that receives data signals from the sensors for further data processing and detects the obstacles around the user up to $1000 \mathrm{~cm}$.A pi camera is used to recognize the person in front of the visually challenged according to the database list .If the person is unknown, then it asks you to store in our data base or not then if we press the push-button then it starts taking the picture of person by using front_facial.xml file so that it can takes the perfect face images using open $\mathrm{cv}$ xml file and through python code. After taking the images it asks you to give the name of the person then mike get activated and then by using google speech to text module it converts the voice input to text and it is saved in a file with person name and id. This device supports to recognize people easily. It is of low cost and low power consumption device.
\end{abstract}

Keywords: Raspberry pi 3, ultrasonic sensors, pi camera, earphones, glasses, visually impaired.

\section{INTRODUCTION}

Eyes play a vital role in the life of human beings as they have the ability to receive and process visual detail to the brain. We perceive that $83 \%$ of information from the environment is obtained through the eyes. There are various disabilities, in which blindness is one among them, in this a person needs to face several problems despite a variety of technological advancements. Blindness is defined as a state of condition where the individual is not capable of seeing and has no light perception. Blindness also refers to those who have low vision that they use other senses as vision substitution skills. Thus, the visually impaired considers the person who has total vision loss or partial vision loss. By considering these factors we tried to reduce the problem mentioned above, and we designed a wearable device called smart glasses that is lighter in weight, cost effective, and not complex in structure, it is easy for visually challenged to carry. Some of

them have many new features but are expensive, so most visually challenged people cannot afford these types of devices. Our proposed device may not be attractive-looking, but our focus was to reach financially weaker visually challenged persons, so they can use it and benefit from it. This proposed device has certain features that are helpful for users in moving around their surroundings independently. Our smart glasses can help visually impaired people to recognise the person in front of the user and learn about obstacles a head. This will be enabled by face recognition and obstacle detection features. Using face recognition technique, the device will identify classmates and relatives by giving some identity or name for new people. If the same person appears in front of the user then the name of a person is given as a message on earphone. The face is playing an important role in conveying identity and emotions.

\section{OVERVIEW OF SYSTEM}

The proposed design uses four components. (1)Raspberry pi 3,(2)Ultrasonic sensor,(3)Pi camera,(4)Earphones .The Ultrasonic sensor has four pins VCC, Trig ,Echo, GND .This ultrasonic sensor is used to detect the object by sending a short ultrasonic burst and then listening for the echo .The Raspberry pi is connected to the ultrasonic sensor that calculates the distance from the object based on the time the echo took to come back .And we generate the output. The output which is in text form is converted to the audio format, which is then relayed to the visually impaired using an Earphone. The pi camera is a video camera that feeds or streams its image in real time. 


\section{LITERATURE SURVEY}

According to reference paper [1] they have presented an architecture for facial and currency identification at people with visual disabilities. To add more advantages for this system we are designing a model with obstacle detection by using the ultrasonic sensors. By using this ultrasonic sensor we can avoid the obstacles in the path and can walk more confidently in the society. According to reference paper [2] the shoes sync with a user phone google maps allows the shoe to keep track of where they are going. Once you have selected an destination and chosen a route, you can tuck your phone away and run along with the left are right in the direction the shoe is buzzing. The main disadvantage is the shoes are of more cost and the processing must be done on the mobile phone and it also detects the objects but not recognise for blind. In this paper we are going detect the object and recognise the object and give the information to the visually challenged using earphones.

\section{BLOCK DIAGRAM}

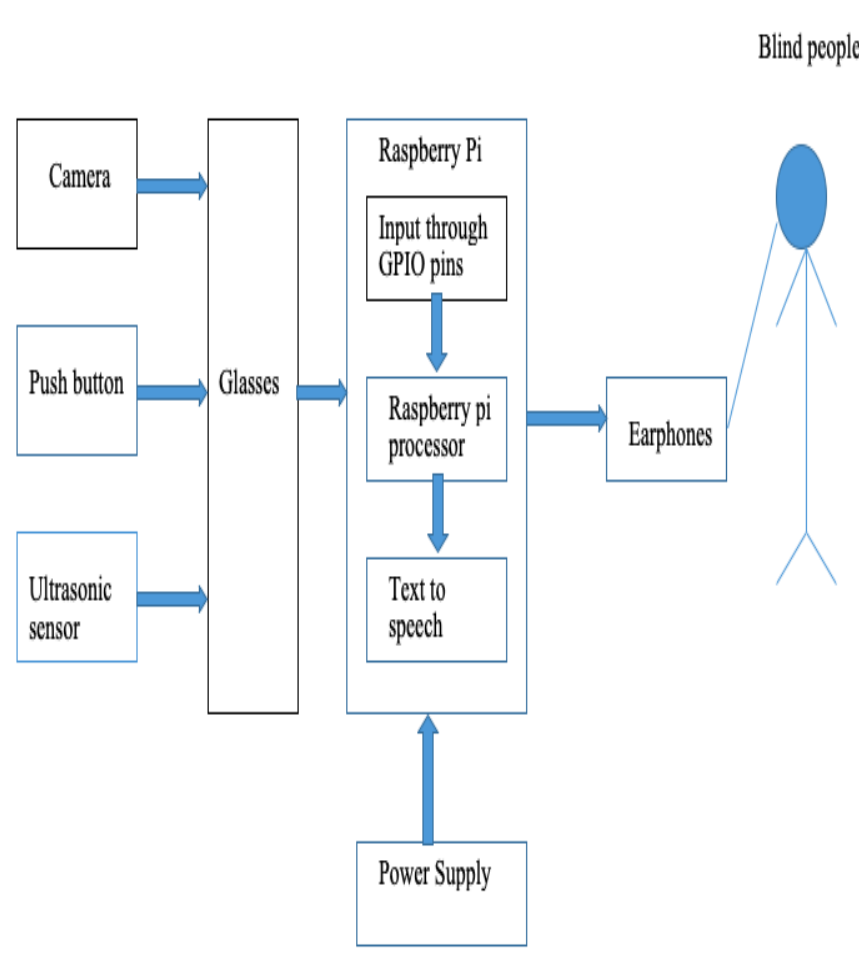

\section{A. Raspberry Pi:}

It is a small sized single board, low cost computer input is taken from the GPIO pins, and it is attached to LEDs, switches and other devices. In our design, we connect the GPIO pins to the ultrasonic sensors. It requires a power source of $5 \mathrm{~V}$ to be operational and we have to insert a Micro Memory SD card in it, which acts as its permanent memory. For our design Raspberry Pi 1 Model B+ is used. It contains 4 USB ports, a HDMI port, an audio jack port and an Ethernet port. The Ethernet port helps the device connect to the Internet and install required driver APIs. It has a $700 \mathrm{MHz}$ single core processor and supports programming languages such as Python, Java, C, and C++ etc. It has the ability to interact with the outside world and has been used in real time applications.

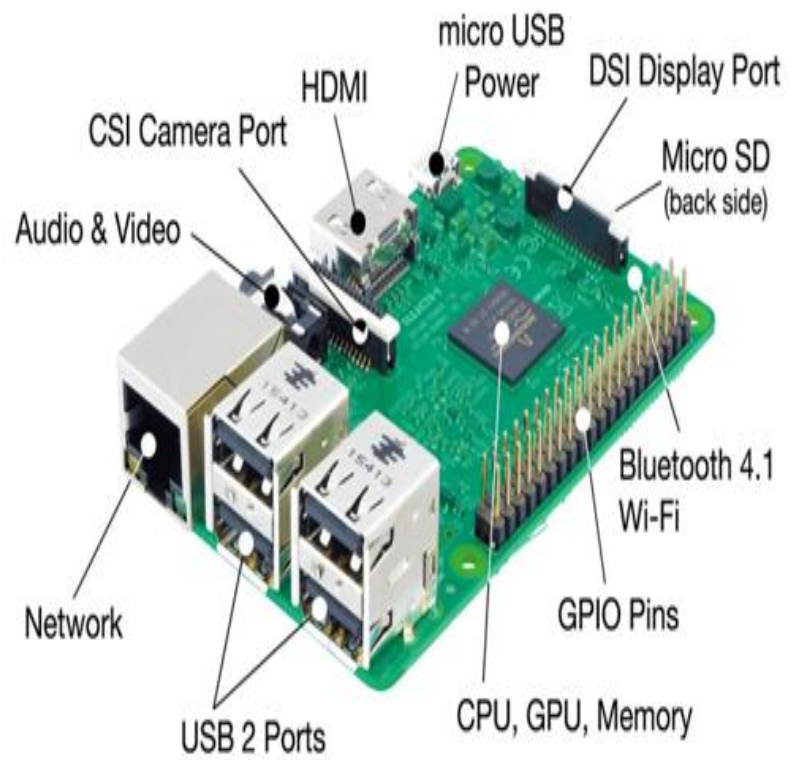

Ultrasonic Sensor:

Raspberry Pi-3 Model B

The Ultrasonic Sensors belong to a set of sensors that emit ultrasound i.e. sound of frequency. A basic ultrasonic sensor consists of one or more ultrasonic transmitters (basically speakers), a receiver, and a control circuit. The transmitters produce high frequency ultrasonic sound, which bounces any nearby solid objects. A bit of that ultrasonic noise is reflected and detected by the receiver on the sensor. Now, control signal is used to process that return signal to calculate the time difference between the signal being transmitted and receiver. The ultrasonic sensor then gives a brief of $40 \mathrm{kHz}$ ultrasonic burst signal. This burst signal travels through the air and hits an object and then reflects back to the sensor giving an output pulse. This output pulse is captured by Raspberry Pi. By using this time taken by the pulse to return back, we calculate the distance from the obstacle. The ultrasonic sensor consists of four pins: (1) VCC, (2) Trigger, (3) Echo and (4) Ground VCC - It is used to provide the power to sensors, typically with $+5 \mathrm{v}$.

Trigger (Trig) - It is an input pin. This pin is used to trigger the ultrasonic sound pulse Echo - It receives the Output Pulse i.e. the echo from the object detected.

Ground (GND) - It connects the sensor to the ground.

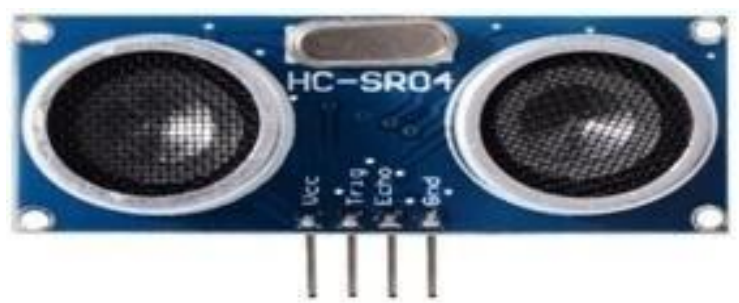




\section{B. Camera Module:}

The Raspberry Pi camera module is mainly used for taking highdefinition video and stills photographs. It has a $15 \mathrm{~cm}$ ribbon cable which is attached to the CSI port on the Raspberry Pi. It is very popular in the face recognition system . 5 images for training and 10 images for recognition are captured using pi camera.

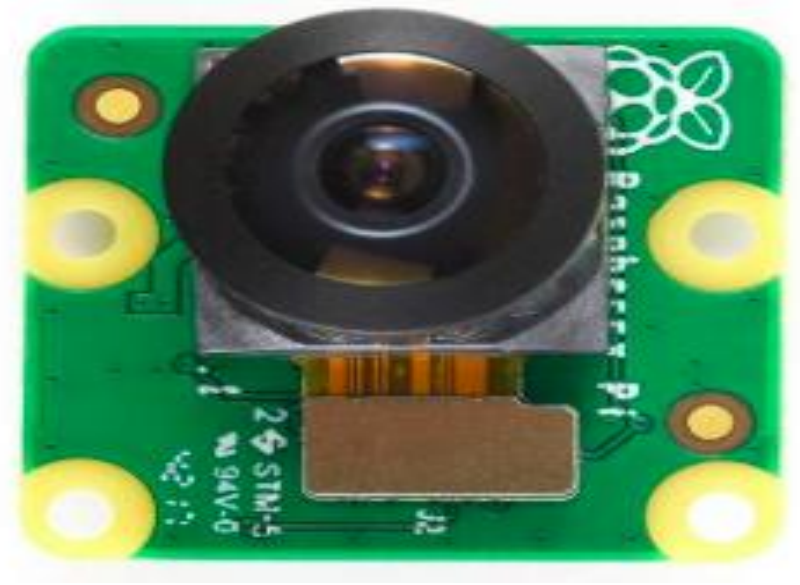

\section{Push Button:}

Push buttons allow us to make any particular connection only when we press the button. Simply, it makes the circuit connected when pressed and breaks when released. It is a simple device and it can be connected to any devices like Arduino and raspberry pi. A push button in simple form it consists of four terminals. The terminals 1 and 2 are connected internally with each other. So, even though you have four terminals, technically you have only two terminals to use.

\section{Ear phones:}

It is the main requirement for users to receive audio messages. It is used to make the visually impaired person aware of the obstacles that are present around them by telling the direction and distance from the obstacle. It is better than a buzzer since, it provides more accurate results and is more perceptive, thereby, helping the person to react more easily.

\section{E. Power Supply:}

This system requires a $5 \mathrm{~V}$ power supply but raspberry pi can work without the main connection by using power bank that can be connected to pi using a battery, portable charger, micro USB or a rectifier as the input power source.

\section{OBSTACLE DETECTION AND DISTANCE CALCULATION}

Obstacle Detection:

Ultrasonic sensors are used for obstacle detection. Distance between the obstacle and the visually impaired people are calculated using ultrasonic sensors. ultrasonic sensors are used in pairs as transceivers i.e. signals will be sent and received by using a single sensor. Eight $40 \mathrm{kHz}$ pulse will be produced by transmitter and this pulse after hitting the obstacle is received back at the Receiver. The ultrasonic sensor works on the principle of sonar. It records the time taken by the emitted pulse to return back at the receiver end. Our algorithm implemented in Python programming language is deployed on Raspberry $\mathrm{Pi}$. This algorithm is used to calculate the distance between the obstacle and the person, by recording the time interval between the pulse sent and pulse received. In this design we use one ultrasonic sensor, which help the person to find any obstacle in left, right or front.

Distance Detection:

To find the distance between the obstacle and the person, we use Distance Formula:

distance $=$ speed $*$ time

$\mathrm{OD}=\{[$ Speed of Sound $*$ Time Taken $] / 2\}$ Where,

OD: Distance between an obstacle and the person in meters.

Speed of Sound: We take speed of sound as 343 meter/sec.

Time Taken: It is the time interval between the pulse emitted and the pulse received.

time taken by the pulse is twice the distance travelled, we divide the equation by 2 .

\section{FACE RECOGNITION}

Face recognition technology shows an open door for visually challenged people to overcome these challenging tasks. An individual is identified by his/her face. We know that the most crucial part of human being is face, used for differentiating a person from another. Each face has different features. So, face recognition plays an important role in human behavior. Face Recognition is defined as a computer vision technique which has the ability of identifying or confirming a user from a digital image or a video frame or a video source or from a surveillance camera. They are compared with a stored database. There are various strategies in which facial recognition systems work, however, they work by comparing selected facial features from given image with faces within a Database. It will identify a person by analyzing patterns based on the person's facial textures and shape. The major challenges of the proposed technique include (a) Face Detection (b) Training the new face images (c) Face recognition, under various conditions like lighting, (d) database (e) voice input/output (f) tagging facility. Face detection is used on many places nowadays especially the websites hosting images.

\section{WORKING}

Step1:

Firstly, whenever the device get activated means giving input voltage then firstly ultrasonic sensor takes the reading of the object. This ultrasonic sensor contains one transducer and trans receiver. Transducer releases some ultrasonic waves and these ultrasonic waves strike off the object and again bounce back. All these waves are again received by the trans receiver and then based on travelling time and using the time and distance relation we can find the object distance The formula is here: distance $=($ duration $* 0.032) / 2$ 
distance in centi meters.

Step 2:

After getting the object distance, if the distance is less than $30 \mathrm{~cm}$ then camera connected to rpi get activated.

firstly camera takes live video streaming then, it converts into frame rate and it that frame rate compares the features with the .yml file which we have generated of different persons images with there id's and if the features matched then it gives the id number based on that number by using if condition we can get the name of the person and the output of the person's name is given through voice by using python Module ESPEAK.

Step3:

If the person is unknown, then it asks you to store in our data base or not then if we press the push-button then it starts taking the picture of person by using front_facial.xml file so that it can takes the perfect face images using open $\mathrm{CV}$.xml file and through python code. After taking the images it asks you to give the name of the person then mike get activated and then by using google speech to text module it converts the voice input to text and it is saved in a file with person name and id.

Then after that based on images, id number and name of the person through open $\mathrm{cv}$ xml file it takes all the parameters of the images and then trained it itself and gives and .yml file. Based on these.$y m l$ file it identifies the person faces.

\section{RESULT}

The project "A New Method For Recognition And Obstacle Detection For Visually Challenged Using Smart Glasses Powered With Raspberry Pi 3" has been successfully designed and tested. In this project, a wearable devices is built in the form of "smart glasses" used for detecting obstacles in front of the user and warn the user about the presence of obstacles in their path. This proposed system is also useful for recognizing known faces stored according to the database list and adding unknown faces to database list. It is of low cost, easy to carry and best solution to visually impaired people.

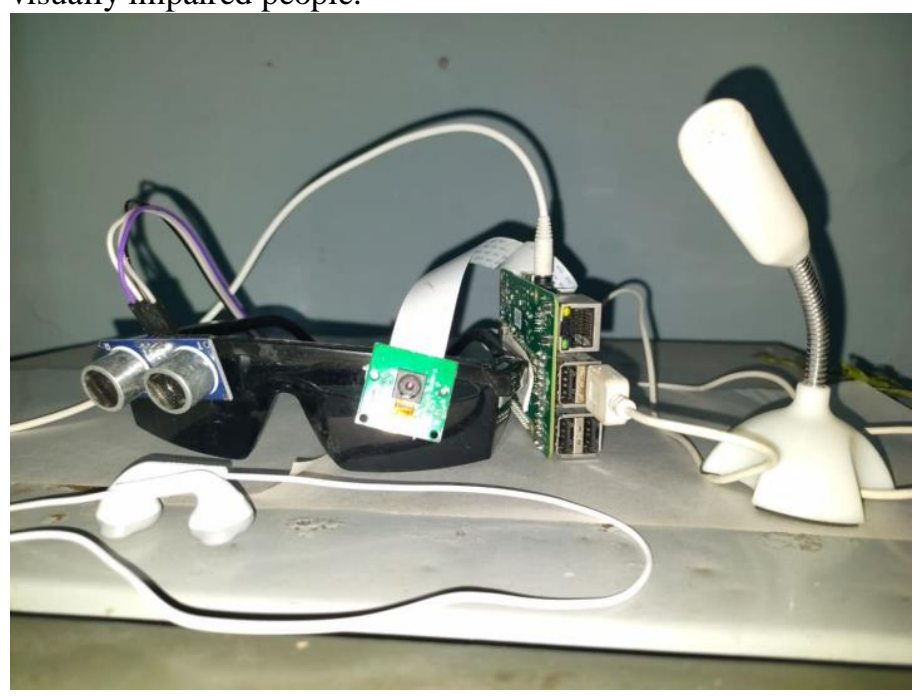

IX. CONCLUSION AND FUTURE WORK

This paper proposes to develop a device within the form a sensible glasses employing a system of Raspberry $\mathrm{Pi}$ and various other components (sensors, earphones, pi camera etc.). it's the subsequent features:

1. A device that helps visually impaired people as walking assistance.

2. Uses sensors to collect information of obstacles.

3. A device which will be used without Internet connectivity.

4. A device that's cost effective, easy to use and portable.

5. A tool that notifies the user about obstacles within the form of speech.

It are often made more compact so on make it easier to hold additionally, if a GPS is installed onto the device, it could also help navigate the person in outdoor environment.

\section{REFERENCES}

[1] Obstacle Detection for Visually Impaired Using Raspberry Pi and Ultrasonic Sensors

Ayush Wattal Dept. of Information Technology JSSATE Noida, India wattal.ayush@gmail.com

Ashutosh Ojha Dept. of Information Technology JSSATE Noida, India ashutoshojha30163@gmail.com

Manoj Kumar Dept. of Information Technology JSSATE Noida, India manojkumar@jssaten.ac.in.

National Conference on Product Design (NCPD 2016), July 2016

[2] Smart Shoe for Visually Impaired Person

Vignesh. ${ }^{1}$,Meghachandra Srinivas Reddy.P ${ }^{2}$, Raja. $^{3}$

,Elamaram.E $\mathrm{E}^{4}$,B.Sudhakar ${ }^{5}$

11vgnshkumar6@gmail.com, ${ }^{2}$ pmcsr999@gmail.com, ${ }^{3}$ rajanirmal1 6@gmail.com, ${ }^{4}$ elamgame@gmail.com

International Journal of Engineering \&Technology, 7 (3.12) (2018) 116-119

[3] Fernández, J. L. carús, R. usamentiaga and R. casado, "Face Recognition And Spoofing Detection System Adapted To Visually-Impaired People"- IEEE Latin America Transactions, vol. 14, no. 2, feb. 2016

[4] A. Carullo and M. Parvis, "An ultrasonic sensor for distance measurement in automotive applications," in IEEE Sensors Journal, vol. 1, no. 2, pp. 143-, Aug 2001.

[5] V. Magori, "Ultrasonic sensors in air," Ultrasonics Symposium, 1994. Proceedings., 1994 IEEE, Cannes, France, 1994, pp. 471-481 vol.1.

[6] A. A. Nada, M. A. Fakhr and A. F. Seddik, "Assistive infrared sensor based smart stick for blind people," Science and Information Conference (SAI), 2015, London, 2015, pp. 11491154.

[7] Z. O. Abu-Faraj, E. Jabbour, P. Ibrahim and A. Ghaoui, "Design and development of a prototype rehabilitative shoes and spectacles for the blind," Biomedical Engineering and Informatics (BMEI), $20125^{\text {th }}$ International Conference on, Chongqing, 2012, pp. 795-799.

[8] K. Audenaert, H. Peremans, Y. Kawahara and J. Van Campenhout, "Accurate ranging of multiple objects using ultrasonic sensors," Robotics and Automation, 1992. Proceedings., 1992 IEEE International Conference on, Nice, 1992, pp. 1733-1738 vol.2.

[9] Liton chandra paul1, Abdulla al sumam2 electronics and telecommunication engineering1,2 rajshahi university of engineering and technology," Face Recognition Using Principal 
Component Analysis Method"-International Journal Of Advanced Research in Computer Engineering \& Technology (IJARCET)volume 1, issue 9, november 2012-

[10] "Object Recognition For Blind People Based on Features Extraction" IEEE Ipas'14: International Image Processing Applications And Systems Conference 2014

[11] Viola, p., \& Jones, m. (2001). "Rapid Object Detection Using a boosted cascade of Simple Features". In Computer Vision And Pattern Recognition, 2001. Cvpr 2001. proceedings of the 2001 IEEE computer society conference on (vol. 1, pp. i-511). IEEE. [12] Huang, G. B., ramesh, m., Berg, t., \& learned-miller, e. (2007). labeled faces in the wild: "A Database For Studying Face Recognition In UnconstrainedEnvironments" (vol. 1, no. 2, p. 3). technical report 07-49, university of massachusetts, amherst.

[13] Vipin kumar jain, Dr. ritu vijay, "Indian Currency Denomination Identification Using Image Processing Technique", (IJCSIT) international journal of computer science $\&$ information technologies.

[14] Pascolini D, Mariotti SPM. Global estimates of visual impairment: 2010. British Journal Ophthalmology Online First published December 1, 2011 as 10.1136/bjophthalmol-2011300539.

[15] A. Helal, S. E. Moore and B. Ramachandran, "Drishti: an integrated navigation system for visually impaired and disabled," Wearable Computers, 2001. Proceedings. Fifth International Symposium on, Zurich, 2001, pp. 149-156.

[16] Dakopoulos and N. G. Bourbakis, "Wearable Obstacle Avoidance Electronic Travel Aids for Blind: A Survey," in IEEE Transactions on Systems, Man, and Cybernetics, Part C (Applications and Reviews), vol. 40, no. 1, pp. 25-35, Jan. 2010. 